\title{
Oscillations in First-Order, Continuous-Time Systems via Time-Delay Feedback
}

\author{
Abimael Salcedo $(\mathbb{D}$ and Joaquin Alvarez \\ Scientific Research and Advanced Studies Center of Ensenada (CICESE), Mexico \\ Correspondence should be addressed to Abimael Salcedo; asalcedo@cicese.edu.mx
}

Received 18 December 2017; Revised 8 August 2018; Accepted 25 September 2018; Published 18 October 2018

Academic Editor: Arturo Buscarino

Copyright (C) 2018 Abimael Salcedo and Joaquin Alvarez. This is an open access article distributed under the Creative Commons Attribution License, which permits unrestricted use, distribution, and reproduction in any medium, provided the original work is properly cited.

\begin{abstract}
A technique to generate (periodic or nonperiodic) oscillations systematically in first-order, continuous-time systems via a nonlinear function of the state, delayed by a certain time $d$, is proposed. This technique consists in choosing a nonlinear function of the delayed state with some passivity properties, tuning a gain to ensure that all the equilibrium points of the closed-loop system be unstable, and then imposing conditions on the closed-loop system to be semipassive. We include several typical examples to illustrate the effectiveness of the proposed technique, with which we can generate a great variety of chaotic attractors. We also include a physical example built with a simple electronic circuit that, after applying the proposed technique, displays a similar behavior to the logistic map.
\end{abstract}

\section{Introduction}

Control and synchronization of chaos have become an intense field of research since several years ago. Some publications pointed out the impact this field may have in many areas of science and technology [1-8]. Chaos anticontrol, that is, producing chaos in a controlled way in a nonchaotic system, has also attracted attention due to its potential usefulness for some important problems of mechanical, electronic, telecommunications, optical, chemical, or biological systems, among others [9-16].

Some techniques to generate chaos in discrete-time systems was proposed in [17-19]. In those papers the aim is to design a feedback nonlinear controller for a linear system such that the closed-loop system displays a chaotic behavior in the sense of $\mathrm{Li}$ and Yorke [20]. The continuous-time counterpart seems to be, however, a more difficult problem.

Introducing a time-delayed state in the feedback control law to modify the behavior of an oscillatory system has been proposed since some time ago [21]. Traditionally, controllers are designed such that the negative effects of time-delay terms on the system performance are attenuated, even suppressed. On the contrary, a time-delay controller makes use of delays to attain some control objectives, for example, stabilizing unstable periodic orbits embedded in a chaotic attractor [2, $4,5,22,23]$. One of the first papers in this line is due to Pyragas [21]. The method proposed therein uses a control signal proportional to the difference between the measured system output and the same (output) signal delayed by a certain time, $u(x(t))=K[y(t-d)-y(t)]$. As a result, chaos is suppressed and the system oscillates periodically, with a period close to the introduced delay $d$, rendering a control signal with small amplitude.

Time delay has also been proposed to generate oscillations, even in first-order systems, which is the aim of the present paper. In [24-26] some conditions are given such that a continuous system can reproduce period-doubling bifurcations displaying by a map, using a singular perturbation technique for delay systems. To find conditions such that a continuous-time system with an input depending on a delayed state displays a chaotic behavior is, however, a more difficult task, and one must rely on numerical or physical experiments to support the investigation. A very known (numerical) example is given in [27], where it is described how a first-order continuous-time system, controlled with a piecewise linear function of the delayed state, displays chaotic behavior. Some other results following the same idea were presented in [28-31] and in $[32,33]$, where fuzzy techniques 
have been employed. Similarly, a model to generate any number of scrolls from a first-order time-delay system is proposed in [34]. A recent and detailed study of this problem was presented in [35], using Fourier series to analyze the generation of oscillations via time-delay states.

The results mentioned were got from approximate methods or oriented to particular systems and supported on numerical or physical experiments. All these results have contributed to establishing that chaotifying a regular system with a simple function of the delayed state is possible. However, developing formal tools or systematic procedures to produce chaotic behavior is a more involved problem.

In this paper, another technique to produce periodic or nonperiodic, even chaotic oscillations in first-order, continuous-time systems via a (nonlinear) function of the delayed state is proposed. Given a first-order passive system, this technique consists in synthesizing a feedback signal given by a nonlinear function of the delayed state, then tuning a gain to ensure that all the equilibrium points of the closed-loop system be unstable, and imposing conditions on the closedloop system to be semipassive. The main property of this procedure is that it can induce a dynamical behavior to the closed-loop system, similar to the behavior displayed by the map defined by the nonlinear function of the delayed state. If this map shows chaotic behavior, then the closed-loop system can also display this dynamics by tuning a parameter. At present time this tuning must be performed via numerical simulation; however, under some conditions established in this paper, it can be ensured that the reproduction of a dynamic behavior is similar to the map. We include several examples to illustrate the effectiveness of the proposed technique, with which a variety of chaotic attractors can be generated. We also include a physical example built with a simple electronic circuit that, after applying the proposed technique, displays a similar behavior to the logistic map.

\section{Preliminaries}

Let us consider a first-order, continuous-time system given by

$$
\dot{x}(t)=f(x(t))+u(t), \quad t \geq 0,
$$

where $(x, u) \in \mathbb{R} \times \mathbb{R}, x$ is the state, $u$ is the input, and $f$ : $\mathbb{R} \longrightarrow \mathbb{R}$ is smooth. Suppose the input is set to

$$
u(x(t))=\gamma(x(t), x(t-1))
$$

where $\gamma: \mathbb{R}^{2} \longrightarrow \mathbb{R}$ is a function such that the delay differential equation

$$
\begin{aligned}
& \dot{x}(t)=f(x(t))+\gamma(x(t), x(t-1)), \quad t \geq 0, \\
& x(t)=x_{0}(t) \quad \text { for } t \in[-1,0]
\end{aligned}
$$

has a solution. Note that, without loss of generality, we can assume a unit time delay. A different delay $d$ can be used, but it can be transformed to unity by a time scaling $\tau=t / d$.
Now consider the so-called "pure-shift" system [36]

$$
\begin{aligned}
& y(t)=\varphi(y(t-1), \theta), \quad t \geq 0, \\
& y(t)=y_{0}(t) \quad \text { for } t \in[-1,0),
\end{aligned}
$$

where $\varphi: \mathscr{R} \times \mathbb{R} \longrightarrow \mathscr{R}, \mathscr{R}$ is a subinterval of $\mathbb{R}$, and $\theta$ is a parameter whose value determines the behavior of this system. If the initial condition ("initial seed") is a constant, $y_{0}(t)=c \in \mathscr{R}$ for $t \in[-1,0)$, then the solution of this system will be

$$
y(t)=\varphi^{k}(c, \theta), \quad k-1 \leq t<k,
$$

where $k \geq 0$ is an integer. Then this system is related to the discrete system

$$
\begin{aligned}
z(k+1) & =\varphi(z(k), \theta), \\
z(0) & =c,
\end{aligned}
$$

in such a way that $y(t)=z(k)$ at time instants $t=k$. Therefore, the solutions of the previous systems (4) and (6) coincide at $t=k$. This means that any dynamical behavior displayed by the discrete-time system (6) will be displayed also by the continuous-time, pure-shift system (4).

An interesting problem is to investigate how a dynamical behavior, similar to that of system (4), can be induced in the closed-loop system (3). To this aim, consider a function $\gamma(2)$ given by

$$
\gamma\left(x, x_{d}\right)=-K\left[x-\varphi\left(x_{d}, \theta\right)\right]
$$

where $K>0$ and $x_{d}$ is the delayed state; that is, $x_{d}(t)=x(t-$ 1 ), leading to the closed-loop system (from (3))

$$
\dot{x}=f(x)-K\left[x-\varphi\left(x_{d}, \theta\right)\right] .
$$

Define $\epsilon=1 / K$; hence system (8) is also described by

$$
\epsilon \dot{x}=\epsilon f(x)-\left[x-\varphi\left(x_{d}, \theta\right)\right] .
$$

Systems with this form have been investigated since some time ago, and several conditions have been given for system (9) to display periodic oscillations and period-doubling bifurcations when the map $\varphi$ undergoes this dynamical phenomena for $\epsilon$ small enough [24, 25, 37]. This behavior gives rise to wonder if there exist some values for $\epsilon$ and $\theta$ such that other kinds of oscillatory behavior can be displayed by this system, behavior that will be clearly related to system (4) and, in consequence, to system (6). This fact will be analyzed later, after establishing some conditions on the equilibrium points of system (8).

\section{Equilibrium Points}

In this section we discuss the relation between the equilibrium points of the delay system (8) and the fixed points of the discrete system (6). These solutions are the simplest dynamical behavior a dynamical system can display. Also, we discuss some conditions for an equilibrium point be stable, which will be used in the next section. 
Lemma 1. Suppose the function $f$ of system (8) is differentiable. Suppose also that, for a given $\theta, \bar{x}_{0}$ is a fixed point of $\varphi(x, \theta)$ and the derivative of this map is defined at $\left(\bar{x}_{0}, \theta\right)$. If $\varphi^{\prime}\left(\bar{x}_{0}, \theta\right) \neq 1$, then there is an equilibrium point $\bar{x}_{\epsilon}$ of system (8), arbitrarily close to the fixed point $\bar{x}_{0}$ of system (6), for $K$ large enough. Furthermore, this equilibrium point $\bar{x}_{\epsilon}$ will be locally, asymptotically stable if, and only if, the next three conditions are all satisfied:

(LS1) $K>f^{\prime}\left(\bar{x}_{\epsilon}\right)-1$,

(LS2) $K\left[1-\varphi^{\prime}\left(\bar{x}_{\epsilon}, \theta\right)\right]>f^{\prime}\left(\bar{x}_{\epsilon}\right)$,
(a) if $K \neq f^{\prime}\left(\bar{x}_{\epsilon}\right)$, there exists $\omega \in(0, \pi), \omega \neq \pi / 2$, that satisfies $\omega+K \varphi^{\prime}\left(\bar{x}_{\epsilon}, \theta\right) \sin \omega>0$, or
(b) if $K=f^{\prime}\left(\bar{x}_{\epsilon}, \theta\right)$, then $\pi / 2+K \varphi^{\prime}\left(\bar{x}_{\epsilon}, \theta\right)>0$.

Proof. An equilibrium point of system (8) is a number $\bar{x}_{\epsilon}$ such that

$$
0=\epsilon f\left(\bar{x}_{\epsilon}\right)-\bar{x}_{\epsilon}+\varphi\left(\bar{x}_{\epsilon}, \theta\right):=\psi\left(\bar{x}_{\epsilon}, \epsilon, \theta\right),
$$

where $\epsilon=1 / K$. That is, given $\epsilon$ and $\theta$, the equilibrium points are the roots of $\psi(\cdot, \epsilon, \theta)$. Because $\bar{x}_{0}$ is a fixed point of the map $\varphi$, then $\psi\left(\bar{x}_{0}, 0, \theta\right)=0$. Given that $\varphi^{\prime}$ is defined at $\bar{x}_{0}$, there exists a neighborhood $V$ of $\bar{x}_{0}$ where $\varphi$ is differentiable. Also, because $\varphi^{\prime}\left(\bar{x}_{0}, \theta\right) \neq 1$, then $\partial \psi / \partial \bar{x}_{\epsilon}\left(\bar{x}_{0}, 0, \theta\right) \neq 0$. Hence, the standard implicit function theorem establishes the existence of a $\mathscr{C}^{1}$-function $g: U \longrightarrow \mathbb{R}$, where $U$ is a neighborhood of the origin, with $U \subset V$, such that $g(0)=\bar{x}_{0}$ and $\psi(g(\epsilon), \epsilon, \theta)=$ 0 for all $\epsilon \in U$, so $g(\epsilon)$ is an equilibrium point of (8). In fact, the convergence of the equilibrium point $\bar{x}_{\epsilon}$ of system (8) to the fixed point $\bar{x}_{0}$ of the map $\varphi$ (i.e., a fixed point of system (6)), when $\epsilon$ converges to zero or, equivalently, when $K$ tends to infinity, is uniform, given the smoothness of function $g$.

To analyze the local stability of the equilibrium point $\bar{x}_{\epsilon}$ we use the known fact that the local stability of an equilibrium point of system (8) can be determined by analyzing its linear approximation [38], given by

$$
\dot{v}=a v+b v_{d}
$$

where $a=f^{\prime}\left(\bar{x}_{\epsilon}\right)-K, b=K \varphi^{\prime}\left(\bar{x}_{\epsilon}, \theta\right)$, and $v_{d}(t)=v(t-$ 1). A direct application to the linear approximation (11) of Theorem A.5 of Hale's book [37], which establishes necessary and sufficient conditions for the (asymptotic) stability of this system, shows that conditions (LS1-3) correspond to the three conditions given in [37].

Remark 2. When the inequalities given by conditions (LS1-3) are replaced by the equality, they give place to the so-called $D$ curves, parameterized by $\omega$, discussed in [39]. For $\omega=0$ and $\pi$ they give the stability boundary for system (11) in the $(a, b)-$ plane. When any of these conditions is not fulfilled, then the linear approximation (11) is not asymptotically stable. In particular, it will be unstable if any of these inequalities is inverted, leading to the instability of the corresponding equilibrium point of system (8).
Example 3. Consider a linear system $(f(x)=-\alpha x)$ and the logistic map $(\varphi(x, \theta)=\theta x(1-x))$,

$$
\begin{aligned}
\dot{x} & =f(x)-K\left[x-\varphi\left(x_{d}, \theta\right)\right] \\
& =-\alpha x-K\left[x-\theta x_{d}\left(1-x_{d}\right)\right],
\end{aligned}
$$

where $\alpha>0, K>0, \theta>1$. The system has the equilibrium points $\bar{x}_{1}=0$ and $\bar{x}_{2}=1-(1+\alpha / K) / \theta$. The conditions for local stability are

(1) For $\bar{x}_{1}$ : (a) $K>-\alpha-1$, (b) $K<\alpha /(\theta-1)$, and (c) if $K+\alpha=0$ then $K \theta>-\pi / 2$, or $K \theta>-\omega / \sin \omega$ if $K+\alpha \neq 0$ for some $\omega \in(0, \pi), \omega \neq \pi / 2$.

(2) For $\bar{x}_{2}$ : (a) $K>-\alpha-1$, (b) $K>\alpha /(\theta-1)$, and (c) if $K+\alpha=0$ then $K(2-\theta)>-2 \alpha-\pi / 2$, or $K(2-$ $\theta)>-\omega / \sin \omega-2 \alpha$ if $K+\alpha \neq 0$ for some $\omega \in(0, \pi)$, $\omega \neq \pi / 2$.

\section{A (Semi)passivity Condition}

In this section we recall an important property that will be used to ensure bounded trajectories of the designed system.

Definition 4 (Khalil [40]). Consider a memory-less system given by the time-varying function

$$
y=h(t, u),
$$

where $h: \mathbb{R} \times \mathbb{R}^{m} \longrightarrow \mathbb{R}^{m}$ is piecewise continuous in $t$ and locally Lipschitz in $u$. This system is passive if there exists a function $\phi: \mathbb{R}^{m} \longrightarrow \mathbb{R}^{m}$ such that

$$
u^{T} y \geq u^{T} \phi(u) \geq 0 \quad \forall u \in \mathbb{R}^{m} .
$$

If (14) holds for $u \notin \mathscr{B}_{r}$, where $\mathscr{B}_{r}$ is a ball with radius $r>0$, $0 \in \mathscr{B}_{r}$, then system (13) is called semipassive.

Now consider a dynamical system with the form

$$
\begin{aligned}
& \dot{x}=f(x, u), \\
& y=h(x, u),
\end{aligned}
$$

where $f: \mathbb{R}^{n} \times \mathbb{R}^{m} \longrightarrow \mathbb{R}^{n}$ is locally Lipschitz, $h: \mathbb{R}^{n} \times \mathbb{R}^{m} \longrightarrow$ $\mathbb{R}^{m}$ is continuous, $f(0,0)=0$, and $h(0,0)=0$.

Definition 5 (Khalil [40]). Suppose there exists a nonnegative $\mathscr{C}^{k}$ - function $S(x)$, with $k \geq 1$ and $S(0)=0$, called the storage function, and $a$ continuous function $H$ such that

$$
\begin{aligned}
& u^{T} y \geq \dot{S}+H(x)=\frac{\partial S}{\partial x} f(x, u)+ H(x) \\
& \forall(x, u) \in \mathbb{R}^{n} \times \mathbb{R}^{m}
\end{aligned}
$$

for any bounded input $u$. This system is called

(i) lossless if $H(x) \equiv 0$ and (16) has the form $u^{T} y=$ $(\partial S / \partial x) f(x, u)$

(ii) passive if (16) holds with $H(x) \geq 0$; 
(iii) semipassive if (16) holds with $H(x) \geq \varrho(\|x\|)$ for $x \notin$ $\mathscr{B}_{r}, r \geq 0,0 \in \mathscr{B}_{r}$, and $\varrho$ is a nonnegative function defined for $\|x\| \geq r$, where $\|\cdot\|$ is the Euclidean norm.

The term strictly (semi)passive is used if the inequalities are strict.

Note 6. For a semipassive dynamical system $\dot{x}=f(x, u)$ such that $f(0,0)=0$ and with a (definite) positive storage function $S$, the ball $\mathscr{B}_{r}$ is attractive. Note that, in this case, $u^{T} y \geq \dot{S}+$ $H(x)$ for $\|x\|>r$. Therefore, if $u=0, \dot{S}=(\partial S / \partial x) f(x, 0) \leq$ $-H(x) \leq-\varrho(\|x\|)<0$ for $\|x\|>r$.

Now consider a negative feedback connection of a passive system $\Sigma_{1}$ and a semipassive system $\Sigma_{2}$ in the feedback loop. Suppose that $u_{i}, x_{i}$, and $y_{i}$ are the input, state, and output of system $\Sigma_{i}$, respectively, $i=1,2$. Because $\Sigma_{1}$ is passive, there exist a storage function $S_{1}\left(x_{1}\right)$ and a function $H_{1}\left(x_{1}\right) \geq 0$ such that $u_{1}^{T} y_{1} \geq \dot{S}_{1}+H_{1}\left(x_{1}\right)$, and because $\Sigma_{2}$ is semipassive, there exist a storage function $S_{2}\left(x_{2}\right)$, a positive number $r$, and a function $H_{2}\left(x_{2}\right)$ that is nonnegative for $\left\|x_{2}\right\| \geq r$, such that $u_{2}^{T} y_{2} \geq \dot{S}_{2}+H_{2}\left(x_{2}\right)$ for $\left\|x_{2}\right\| \geq r$. If $u$ is the input for the connected systems and $y$ the output, then $u=u_{1}+y_{2}, u_{2}=y_{1}$, and $y=y_{1}$. Therefore

$$
u^{T} y \geq \dot{S}_{1}+H_{1}\left(x_{1}\right)+\dot{S}_{2}+H_{2}\left(x_{2}\right)=\dot{S}+H(x),
$$

where $x=\left(x_{1}^{T}, x_{2}^{T}\right)^{T}, S=S_{1}+S_{2}, H(x)=H_{1}\left(x_{1}\right)+H_{2}\left(x_{2}\right)$, and $H(x) \geq 0$ if $\|x\| \geq \delta$ for some $0<\delta \leq r$.

In summary, a feedback connection of a passive system with a semipassive one is semipassive. In the same way, the feedback connection of two semipassive systems is semipassive. Note that the state trajectories are bounded because the connection is semipassive.

We are now in position to state the main result.

Theorem 7. Let us consider the first-order system

$$
\begin{aligned}
\Sigma_{1}: \quad \dot{x} & =f(x)+u, \\
y & =x,
\end{aligned}
$$

where $f: \mathbb{R} \longrightarrow \mathbb{R}$ is $\mathscr{C}^{1}$ and $f(0)=0$, with the input signal

$$
u(x)=-K[x-\varphi(x(t-1), \theta)]
$$

with $K>0$, and suppose the following assumptions are satisfied:

(AS1) For any $x \neq 0, x f(x)<0$.

(AS2) For a given $\theta$, the map $\varphi(x, \theta)$ is bounded, $\varphi(0, \theta)=0$, and satisfies that

(a) its derivative $\varphi^{\prime}(\cdot, \theta)$ is defined at any equilibrium point $\bar{x}$ of the closed-loop system (18)-(19), and $\left|\varphi^{\prime}(\bar{x}, \theta)\right|>1$;

(b) there exists two intervals, $J=(a, d)$ and $I=$ $(b, c)$, with $a \leq b<0<c \leq d$ such that $x \varphi(x, \theta)>x^{2}$ for $x \in I \backslash\{0\}, x \varphi(x, \theta) \leq x^{2}$ for $x \notin I$, and $\varphi(x, \theta)=0$ for $x \notin J$.
(AS3) At any equilibrium point $\bar{x}$ of the closed-loop system (18)-(19), the following inequality is satisfied:

(a) if $\varphi^{\prime}(\bar{x}, \theta)>1$, then

$$
K>\frac{f^{\prime}(\bar{x})}{1-\varphi^{\prime}(\bar{x}, \theta)}
$$

(b) if $\varphi^{\prime}(\bar{x}, \theta)<-1$, then there exists $\omega \in(0, \pi)$ such that

$$
K>\frac{-\omega}{\varphi^{\prime}(\bar{x}, \theta) \sin \omega} .
$$

Then there exists a positive number $K_{\min }$ such that the feedback system (18)-(19) exhibits bounded, oscillatory solutions for all $K>K_{\min }$. Depending on the value of gain $K$ and the dynamical behavior defined by the map $\varphi(z, \theta)$, these solutions may be periodic or not periodic.

Proof. First we prove the instability of the equilibrium points of the closed-loop system (18)-(19). For $\varphi^{\prime}(\bar{x}, \theta)>1$ this is easy to see from assumption (AS3a) because inequality (20) is the opposite of assumption (LS2) of Lemma 1 (note that $\left.1-\varphi^{\prime}(\bar{x}, \theta)<0\right)$. For $\varphi^{\prime}(\bar{x}, \theta)<-1$ note that $(21)$ of assumption (AS3b) is the opposite of the inequalities given in the assumption (LS3) of Lemma 1. Therefore, assumptions (AS3a,b) guarantee the instability of the equilibrium points of the closed-loop system, avoiding the state $x$ convergence to a constant.

Now define the system

$$
\Sigma_{2}: \quad v(y)=K\left[y-\varphi\left(y_{d}, \theta\right)\right]
$$

which has input $y$ and output $v$. The closed-loop system (18)-(19) is composed by the negative feedback connection $(u=-v)$ of systems $\Sigma_{1}$ and $\Sigma_{2}$. Hence this connection will be semipassive if one system is passive and the other one is semipassive.

Consider system $\Sigma_{1}(18)$, the storage function $S(x)=$ $x^{2} / 2$, and assumption (AS1). Then $u y=(\dot{x}-f(x)) x=$ $\dot{S}-x f(x)=\dot{S}+H(x)$, where $H(x)=-x f(x)>0$ for $x \neq 0$. By Definition 5 , system $\Sigma_{1}$ is strictly passive.

Now we prove the semipassivity of $\Sigma_{2}$. For this, first consider the related system

$$
\widehat{v}(y)=K[y-\varphi(y, \theta)]
$$

By Assumption (AS2b),

$$
y \widehat{v}=K y[y-\varphi(y, \theta)] \begin{cases}<0, & \forall y \in I \backslash\{0\} ; \\ >0, & \forall y \notin J .\end{cases}
$$

Note that $\varphi\left(y_{d}\right)=\varphi(y+\delta y)$ is a displacement of the map $\varphi$ along the horizontal axis. Note also that $\varphi(y, \theta)=0$ for $y \notin J$ (assumption (AS2b)). In this way, for any $y_{d}=y+$ $\delta y \notin J$ we have $y v=K y^{2}>0$, leading eventually to $y \notin J$, ensuring the semipassivity of the block. Because $y$ and $y_{d}$ are 
not necessarily at the same time outside $J$, the interval where $K y\left(y-\varphi\left(y_{d}, \theta\right)\right)>0$ may be time varying, but the inequality

$$
y v=K y\left[y-\varphi\left(y_{d}, \theta\right)\right]>0
$$

holds for $y$ and $y_{d}$ outside some interval $\widetilde{J}$, and system (22) will be semipassive.

Given that the closed-loop system is composed by a feedback connection of the passive system (18) with the semipassive system (22) via $u=-v$, then the feedback connection (18)-(19) is semipassive, and the solutions of the closed-loop system will be bounded.

In consequence, because the closed-loop system cannot converge to the equilibrium points (they are unstable) and the system trajectories are bounded (due to the semipassivity condition), they must be oscillatory.

To see the existence of a minimal $K$ ensuring this fact, if $\varphi^{\prime}(\bar{x})>1$, then $1-\varphi^{\prime}(\bar{x}, \theta)<0$ and (20) has the form $K>f^{\prime}(\bar{x}) /\left[1-\varphi^{\prime}(\bar{x}, \theta)\right]=K_{1}$. In this case, if $K_{1}$ is positive, we set $K>K_{1}$, and $K>0$ otherwise. On the other hand note that, if $\varphi^{\prime}(\bar{x})<-1,(21)$ has the form $K>-\omega /\left[\varphi^{\prime}(\bar{x}) \sin \omega\right]=$ $K_{2}>0$. Suppose there are $m$ equilibrium points of the closedloop system (18)-(19). Therefore, for each equilibrium point $\bar{x}_{i}, i=1, \ldots, m$, there will be a minimal value of $K$, denoted by $K_{i}$, that makes this point unstable. Hence, to make unstable all the equilibrium points, we choose

$$
K>K_{\min }=\max \left\{K_{i}, i=1, \ldots, m\right\} .
$$

Remark 8. Note that when $x_{d} \notin J$, then $\varphi\left(x_{d}, \theta\right)=0$ and the closed-loop system transforms to $\dot{x}=f(x)-K x$. Because $\dot{x}=f(x)$ is strictly passive and $K>0$, this system is attracted to $x=0$ while $x_{d} \notin J$, but it does not converge to the origin because it is unstable. If at a certain time the state is near to the origin, it will be repelled, eventually reaching the region where it will be attracted again. It is this exchange of "energy" that generates the oscillatory behavior of the closedloop system.

Remark 9. Theorem 7 establishes sufficient conditions for the existence of a minimum value of the gain $K$ that guarantees an oscillatory behavior. However, the generation of nonperiodic or chaotic oscillations depends on the dynamic behavior of the system defined by the map $\varphi(x, \theta)(6)$ via the parameter $\theta$ and should be performed numerically, for values larger than $K_{\min }$. What is sure is that system (18)-(19) will behave similarly to the map (6) for values of $K$ greater than $K_{\min }$.

\section{Examples}

In this section we show some examples to illustrate the proposed technique. In particular, we consider system (18)(19) with $f(x)=-x$ and the map $\varphi$ the logistic, sine, and triangular maps defined in the interval $J=(a, d)$, according to Theorem 7, and zero elsewhere. Hence, the closed-loop system has the form

$$
\begin{aligned}
& \dot{x}=-x-K\left(x-\varphi\left(x_{d}\right)\right), \\
& y=x,
\end{aligned}
$$

where $K>0$ and $x_{d}(t)=x(t-1)$. Note that $x f(x)=-x^{2}<0$ for $x \neq 0$; then assumption (AS1) of Theorem 7 is satisfied.

5.1. The Logistic Map. In this section the Logistic map is given by

$$
\varphi\left(x_{d}\right)= \begin{cases}\mu x_{d}\left(1-x_{d}\right), & x_{d} \in J=\left(0_{-}, 1\right) ; \\ 0, & x_{d} \notin J,\end{cases}
$$

where $\mu>1$ and $0_{-}$is an arbitrary small negative number. We denote the parameter $\theta$ by $\mu$, which is a standard notation for the logistic parameter.

As seen in Section 2, the closed-loop system has two equilibrium points, $\bar{x}_{1}=0$ and $\bar{x}_{2}=1-(1+1 / K) / \mu$. Moreover, $\varphi(x)$ is bounded, $\varphi^{\prime}\left(\bar{x}_{1}\right)=\mu, \varphi^{\prime}\left(\bar{x}_{2}\right)=\mu[-1+$ $2(1+1 / K) / \mu]$ are well defined, $\varphi^{\prime}(x)>1$ if $\mu>1$ for $\bar{x}_{1}$ and $\varphi^{\prime}(x)<-1$ if $\mu>3+2 / K$ for $\bar{x}_{2}$, and (AS2a) is satisfied. For this case, $J=\left(0_{-}, 1\right), I=\left(0, \bar{x}_{2}\right)$, and it is easy to show that assumption (AS2b) is fulfilled.

It is known that the logistic map shows an oscillatory behavior starting from $\mu=3$; hence let us suppose $\mu>3$. Assumption (AS3a) holds for $\bar{x}_{1}$ if $K>1 /(\mu-1)=K_{1}$, and (AS3b) is fulfilled for $\bar{x}_{2}$ if $K>2+\omega / \sin \omega=K_{2}$, for some $\omega \in(0, \pi)$. In fact, the minimum number that satisfies this last inequality is for $\omega=0^{+}$, where $0^{+}$is an arbitrary small positive number, so $K_{2}=3$. Hence, $K_{\min }=\max \{1 /(\mu-1), 3\}=3$ because $\mu>3$; then $K$ must be greater than 3 . This means that the oscillatory behavior of system (27)-(28), with $\mu>3$, starts with $K=3$.

Figure 1 shows several orbits for different values of parameter $K$, with $\mu=4$. Note that the oscillatory behavior starts at $K=3$ (Figure $1(\mathrm{a})$ ), and the system exhibits periodic behavior for $K=5$ (Figure 1(b)), 2-periodic orbits for $K=$ 8 (Figure 1(c)), and an irregular behavior that seems to be chaotic for $K=20$ (Figure 1(d)). Figure 2 summarizes the behavior of this system for $(K, \mu)$ in some region of the plane. The blue zone corresponds to stable equilibrium points, the green zone to periodic behavior, and the red zone to nonperiodic and chaotic dynamics. Note that the chaotic behavior starts at $\mu$-values smaller than 4 , if $K$ is large enough.

Figure 3 shows the maximum Lyapunov exponent as a function of the parameter $K$ and $\mu=4$. Note that, for this value of parameter $\mu$, the maximum Lyapunov exponent is positive for $K>9$, which corresponds well to the $(K, \mu)$ values shown in Figure 2.

Finally, Figure 4 shows the bifurcation diagram and the location of one of the unstable equilibrium points when the parameter $K$ varies in the interval $[4,20]$. Note that the unstable equilibrium $(\bar{x}=1-(\alpha / K+1) / \mu=0.72$ if $K=30)$ converges close to $1-1 / \mu=0.75$ for $K$ large enough. A wide range of dynamical behavior of this system can be seen. Note also that the values for periodic and chaotic behavior correspond well to Figure 3.

5.2. The Sine Map. Now consider the same linear, strictly passive system of the previous section (27) with the sine function $\varphi\left(x_{d}\right)=A \sin \left(x_{d}\right)$, for $x_{d} \in(-\pi, \pi)$, and zero elsewhere. Then $f^{\prime}(x)=-1$ and $\varphi^{\prime}(x)=A \cos x$. 


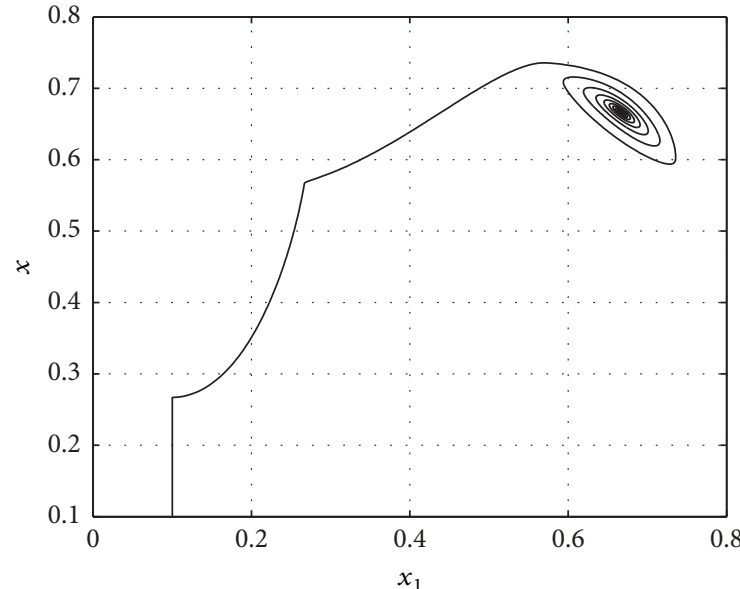

(a) $K=3$

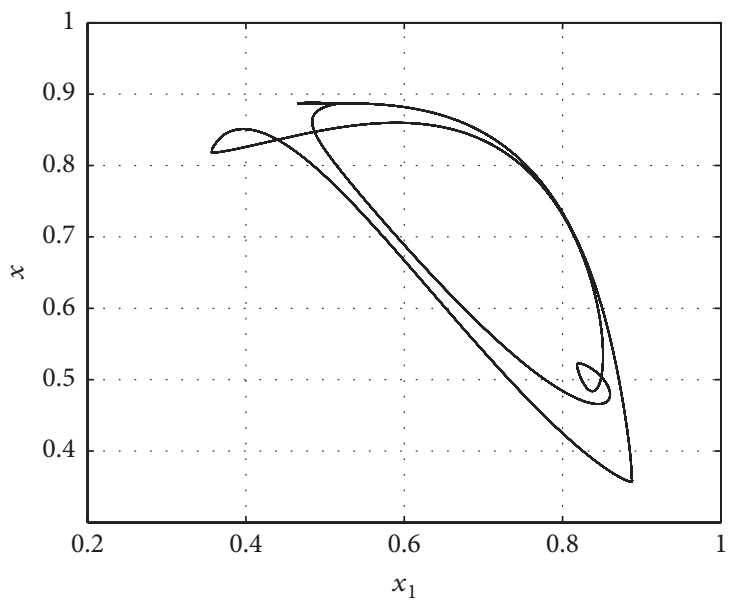

(c) $K=8$

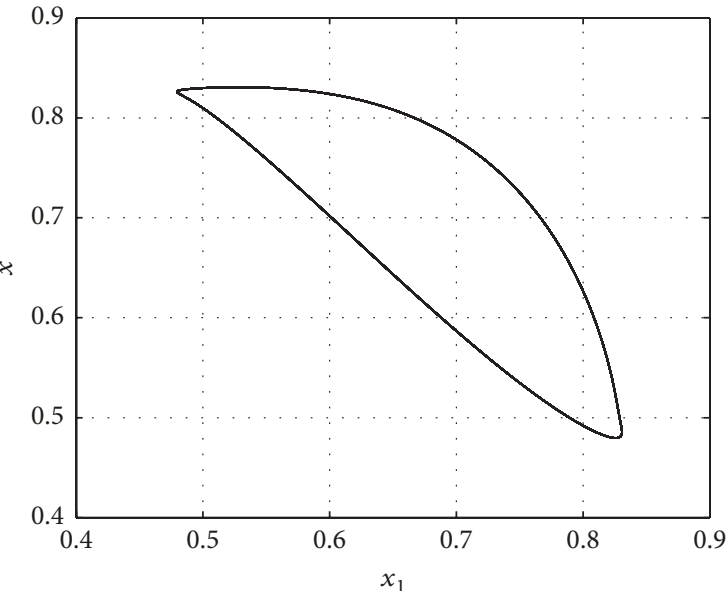

(b) $K=5$

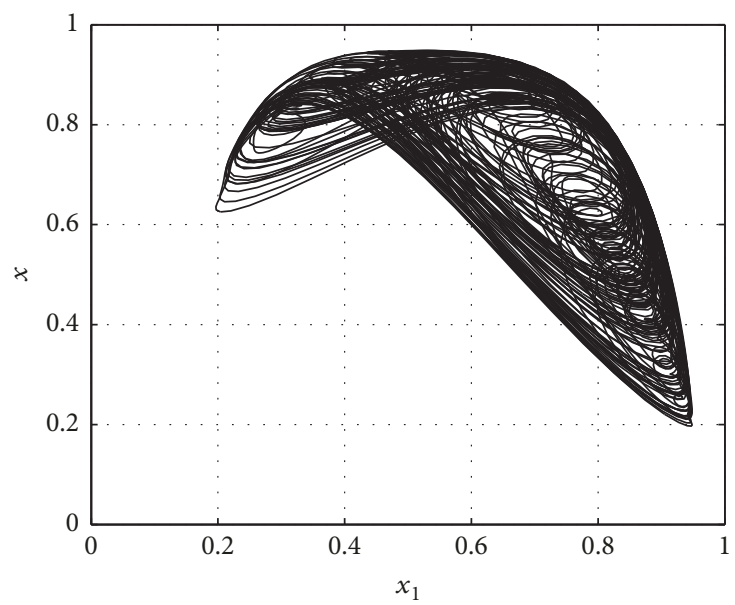

(d) $K=20$

FiguRE 1: Responses of system (27) with the Logistic map (27), for $\mu=4$ and different values of $K$.

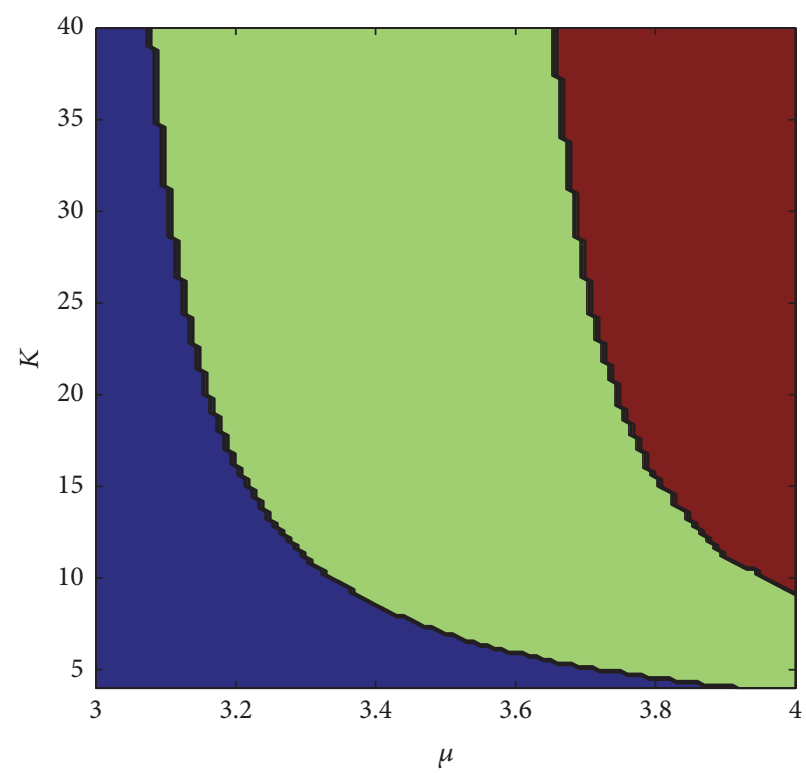

FIGURE 2: Equilibrium (blue), periodic (green), and nonperiodic and chaotic (red) behavior of system (27) with the Logistic map (28) in the $(K, \mu)$-parameter space. 


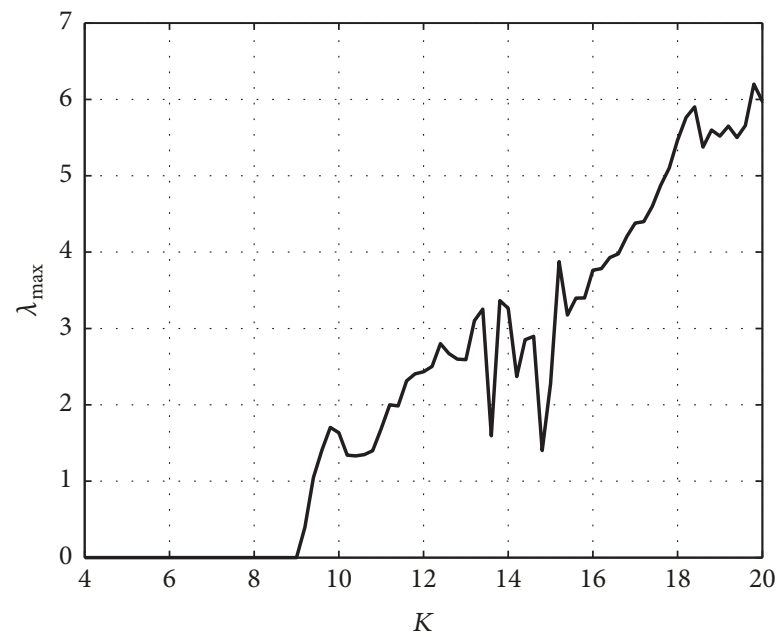

FIgURE 3: Maximum Lyapunov exponent of system (27) with the Logistic map (28) as a function of parameter $K$.

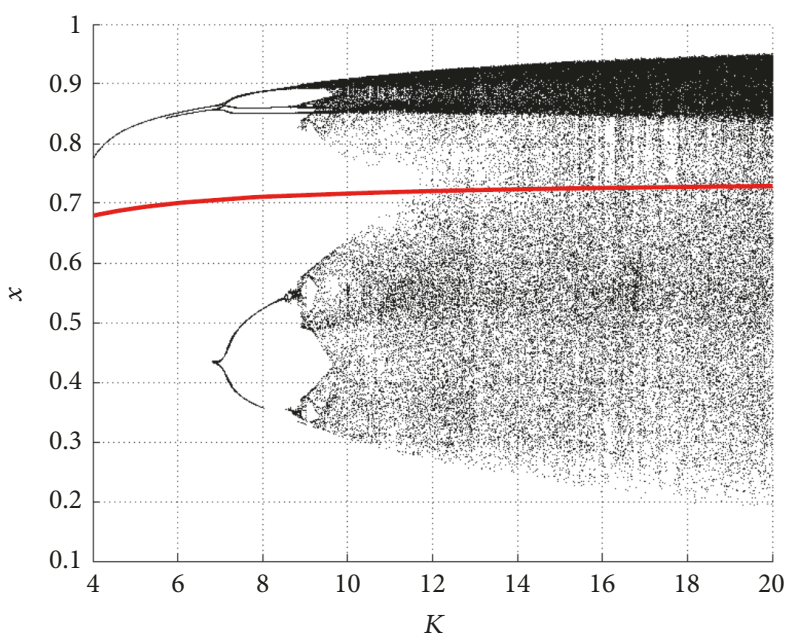

FIGURE 4: Bifurcation diagram and location of the unstable equilibrium point of system (27) with the Logistic map (28).

The origin $\bar{x}_{1}=0$ is an equilibrium point. Since $\varphi^{\prime}(0)=$ $A$, then it can be made greater than one if $A>1$. Therefore, from (20), $K>1 /(A-1)$ makes the origin unstable. Under this condition $(A>1+1 / K)$ the closed-loop system will have two other equilibrium points $\bar{x}_{2} \in(\pi / 2, \pi)$, and $\bar{x}_{3}=-\bar{x}_{2}$, solutions of the equation $A \sin (\bar{x}) / \bar{x}=1+1 / K$. For these last two points, $\varphi^{\prime}(\bar{x})=A \cos (\bar{x})$; then $\varphi^{\prime}(\bar{x})$ can be made less than -1 with $A$ large enough, which will set $\bar{x}_{2}$ in the interval $(\pi / 2, \pi)$ that makes negative $\cos \left(\bar{x}_{2}\right)$. Therefore, (21), rewritten as $K A \cos \left(\bar{x}_{2}\right)<-\omega / \sin \omega$, can be satisfied for some $\omega \in(0, \pi)$. In fact, this condition can be satisfied if $K A>-1 / \cos \left(\bar{x}_{2}\right)$. A similar analysis can be made for $\bar{x}_{3}$, leading to the same condition.

On the other hand

$$
y v=K x\left(x-A \sin \left(x_{d}\right)\right)>0 \quad \text { if } x_{d} \notin(-\pi, \pi),
$$

and all the assumptions established in Theorem 7 hold.
Figure 5 shows some attractors for different values of the gain $K$. When the condition given by the previous theorem is satisfied, Figures $5(\mathrm{~b})-5(\mathrm{~d})$, the system exhibits periodic and chaotic orbits. The $(A-K)$-parameter space shows the region where the system exhibits stable equilibrium points, periodic and chaotic orbits; see Figure 6. Figure 7 shows the bifurcation diagram and location of an unstable equilibrium point of the system with respect to the parameter $K$, with $A=3$, and Figure 8 shows the maximum Lyapunov exponent as a function of parameter $K$. The unstable equilibrium of the closed-loop system converges to the fixed point of map $\varphi(x, A)$ for $K$ large enough.

Other attractors can be obtained by changing the interval where the map $\varphi\left(x_{d}\right)$ is different to zero. For example, with $A=5, K=10, x_{d} \in[-2 \pi, 2 \pi]$, this system displays the orbit shown in Figure 9(a), and if $A=-5, K=10$, and $x_{d} \in$ $[-2 \pi, 2 \pi]$, it displays the orbit shown in Figure 9(b). 


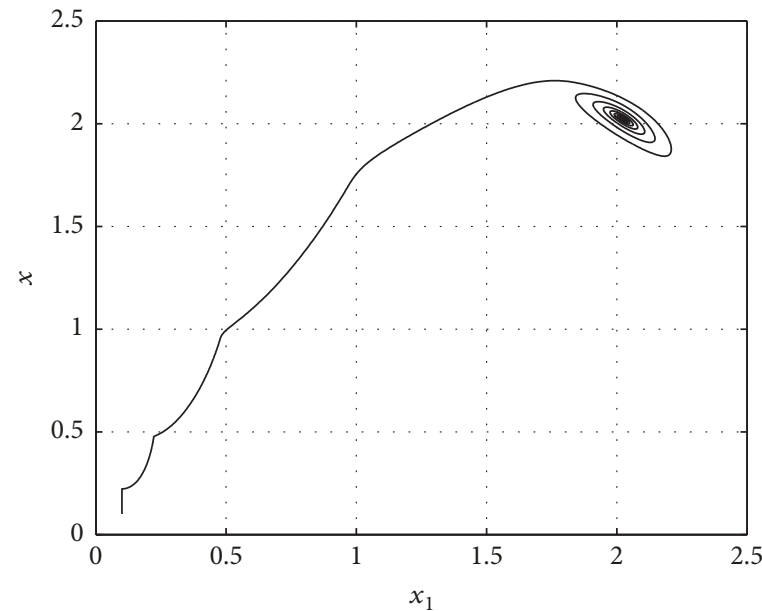

(a) $K=3$

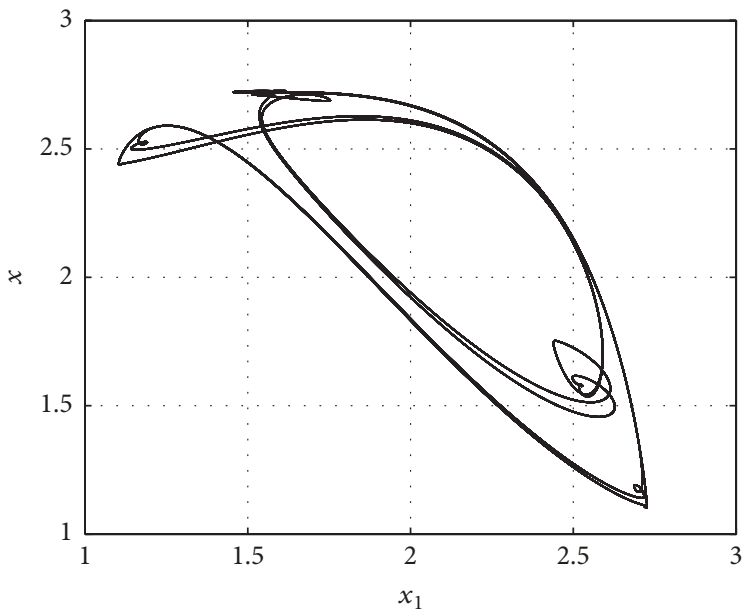

(c) $K=10$

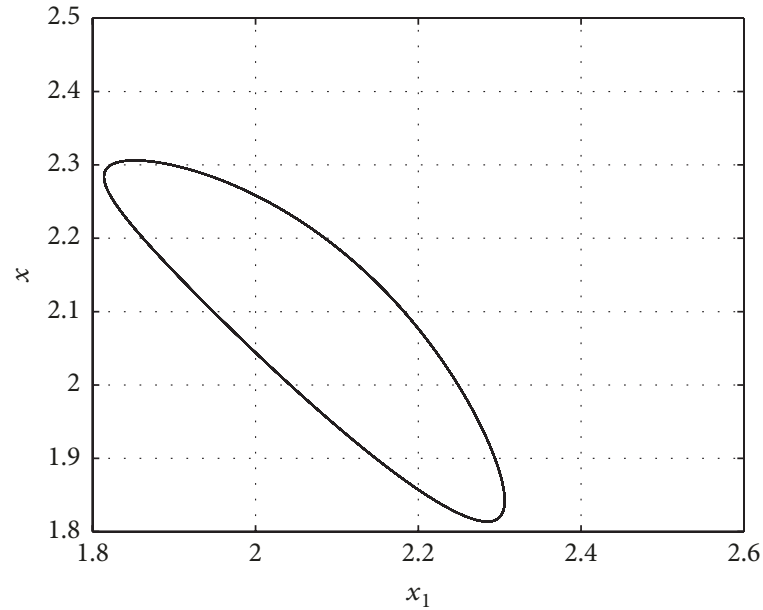

(b) $K=4$

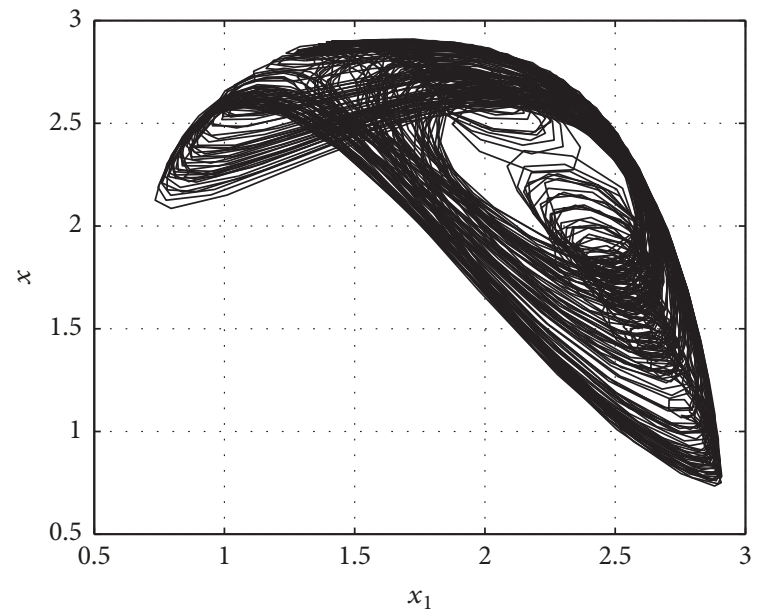

(d) $K=40$

Figure 5: Responses of system (27) with the sine map, $A=3, J=(-\pi, \pi)$, and several values of $K$.

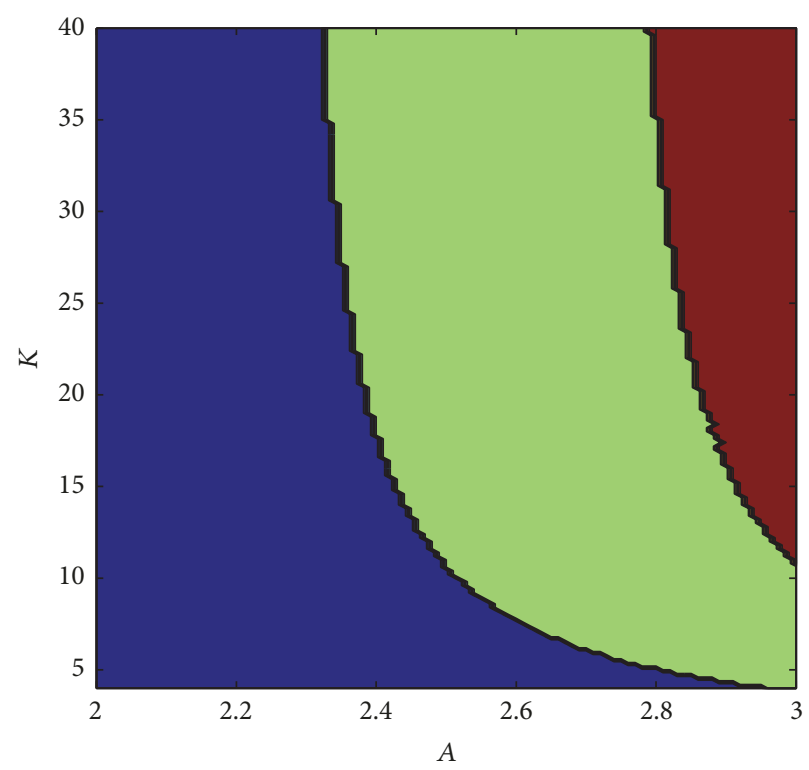

FIGURE 6: Equilibrium (blue), periodic (green), and nonperiodic and chaotic (red) behavior of system (27) with the sine map. 


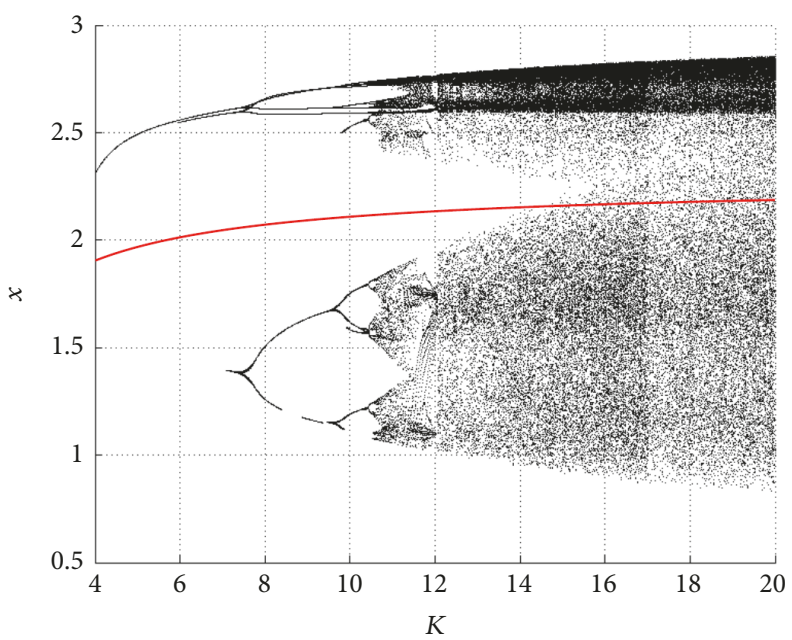

FIGURE 7: Bifurcation diagram and location of an unstable equilibrium point of system (27) with the sine map.

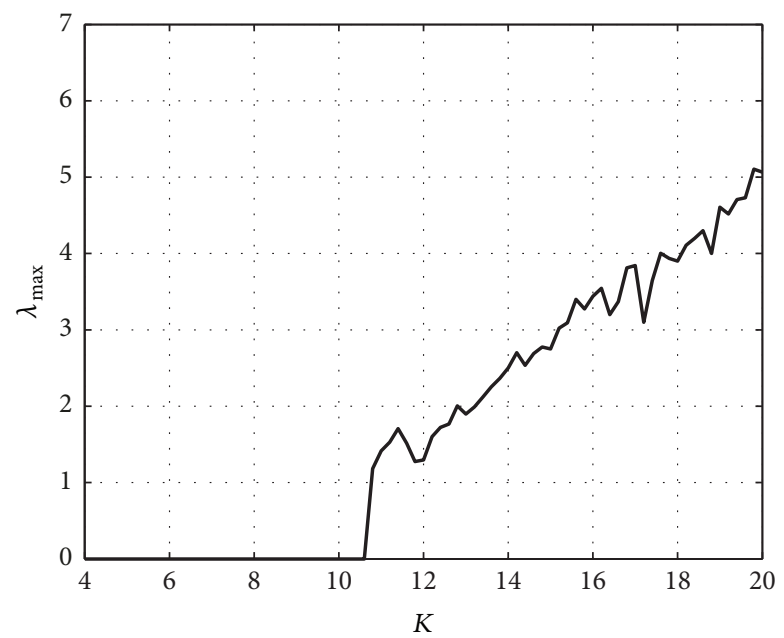

FIGURE 8: Maximum Lyapunov exponent of system (27) with the sine map.

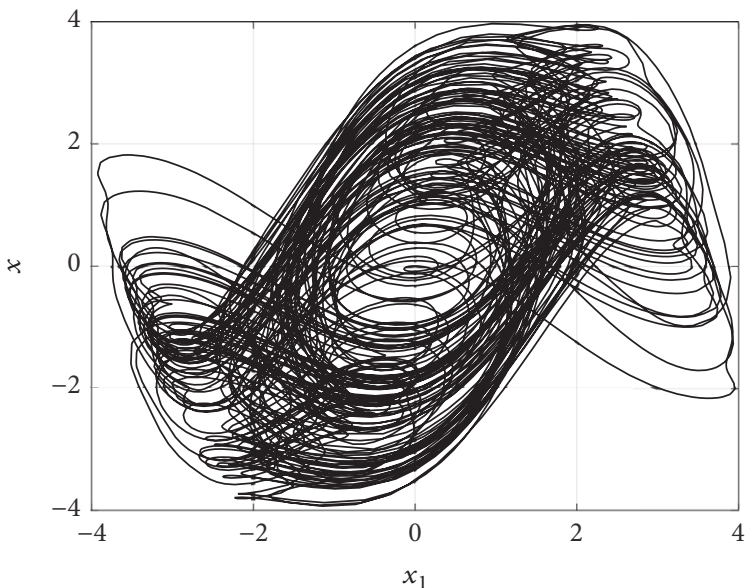

(a)

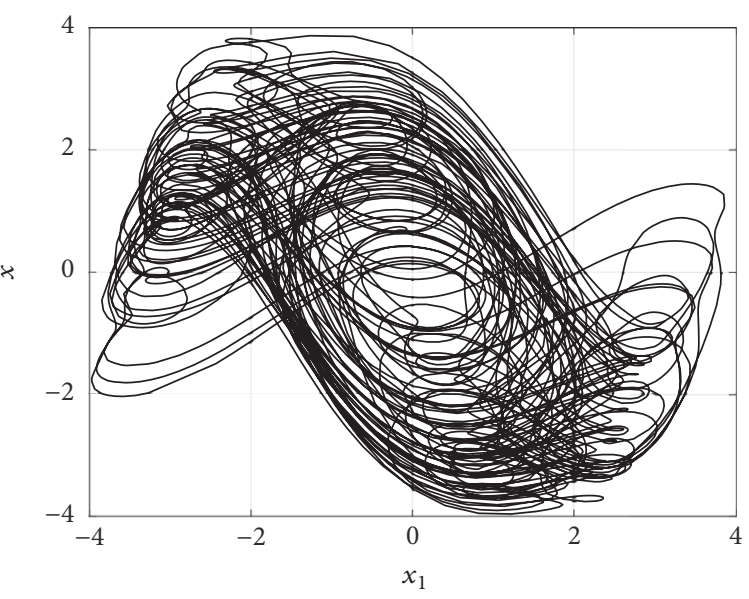

(b)

Figure 9: Attractors of system (27) with the sine map: (a) $A=5, K=10, x_{d} \in[-2 \pi, 2 \pi]$; (b) $A=-5, K=10, x_{d} \in[-2 \pi, 2 \pi]$. 


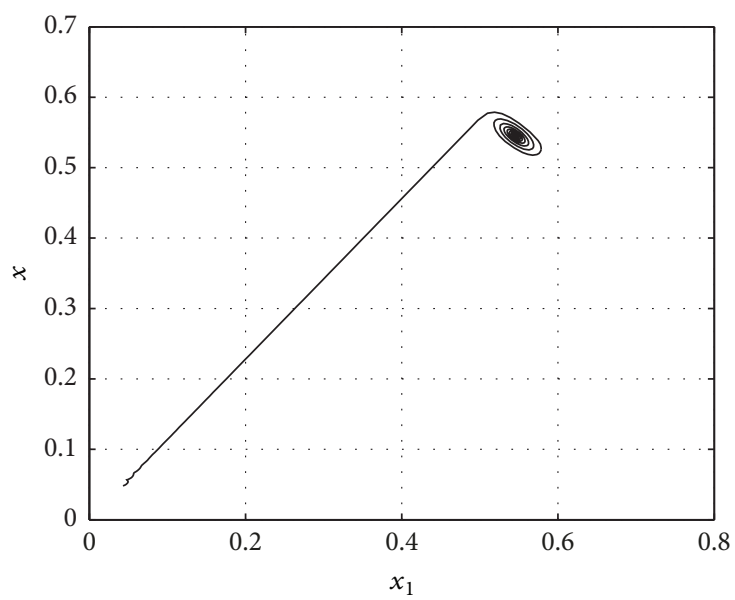

(a) $K=1.5$

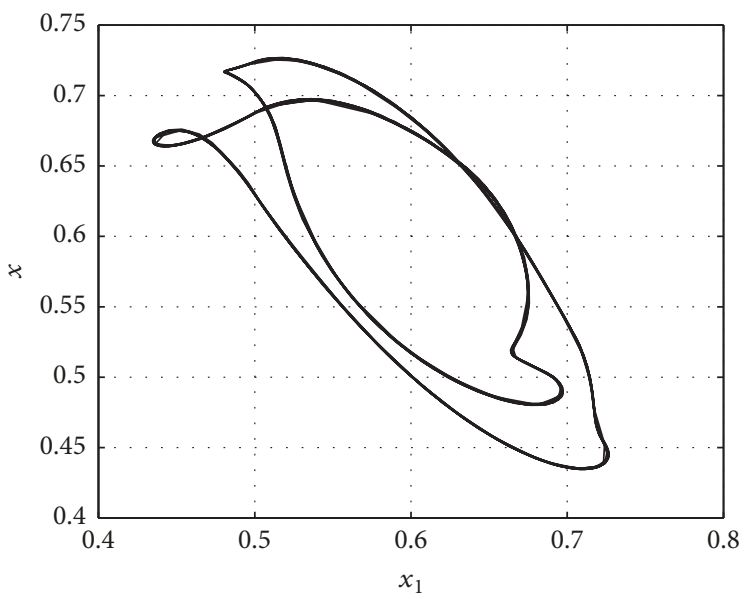

(c) $K=3$

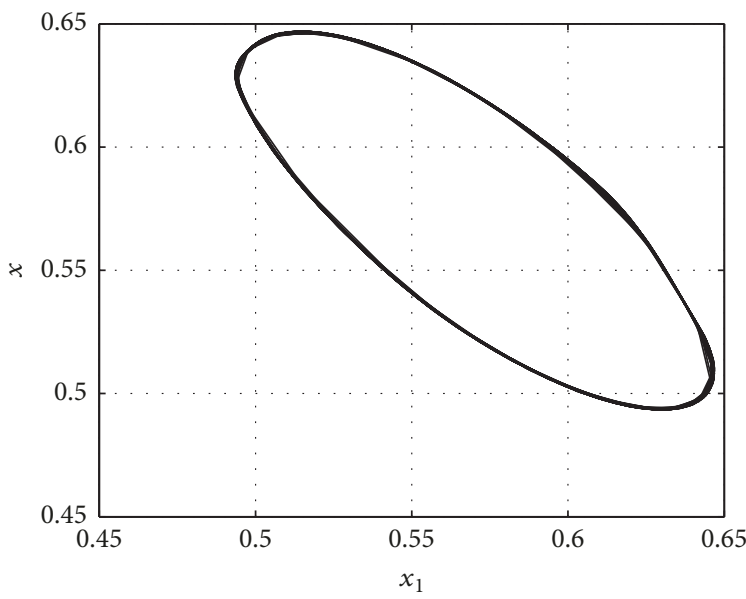

(b) $K=2$

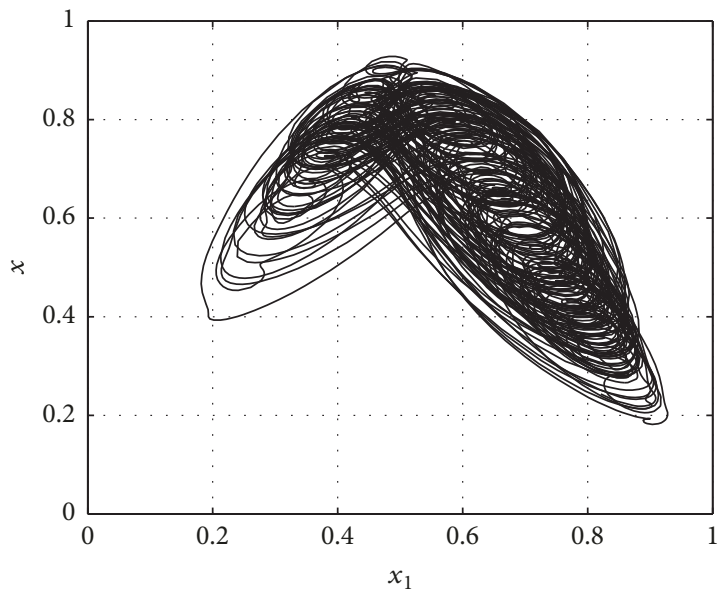

(d) $K=20$

FIGURE 10: Responses of system (27) with the triangular map (30) for $a=2$.

5.3. The Triangle Map. Finally, consider system (27), where

$$
\varphi\left(x_{d}\right)= \begin{cases}a x_{d}, & x_{d} \in(0,0.5) ; \\ a\left(1-x_{d}\right), & x_{d} \in[0.5,1) ; \\ 0, & \text { elsewhere, }\end{cases}
$$

with $1<a \leq 2$. This system is piecewise linear and has two equilibrium points, $\bar{x}_{1}=0$ and $\bar{x}_{2}=1 /(1+(K+1) / K a)$. For both equilibria, $f^{\prime}(\bar{x})=-1$.

Since $\varphi^{\prime}\left(\bar{x}_{1}\right)=a>1$, assumption (AS2) is fulfilled and (AS3a) leads to $K>1 /(a-1)$. Moreover, $\varphi^{\prime}\left(\bar{x}_{2}\right)=-a<-1$; then (AS2) holds and (AS3b) is satisfied if $K>1 / a$ (for $\omega=$ $\left.0_{+}\right)$. Given that $a>1$, then $K_{\min }=1 /(a-1)$.

Figure 10 shows different dynamics of system (27)-(30) for different values of the gain $K$.

This system can display several behaviors like stable equilibrium points, periodic or chaotic orbits. These different dynamics can be viewed in the $a-K$ plane, as shown in Figure 11. Figure 12 shows the largest Lyapunov exponent as function of the parameter $K$, with $a=2$. The bifurcation diagram, shown in Figure 13, has been generated with the same parametric values as the Lyapunov exponent diagram. This diagram shows also an unstable equilibrium point, which converges to the corresponding fixed point of the map, for K large enough.

\section{A Chaotic Circuit}

The dynamic behavior shown in the previous section can also be observed in simple physical systems. In this section we show the physical implementation of a linear, passive circuit with an input signal depending on the delayed state processed by a nonlinear function given by the logistic map. The dynamics of the circuit, shown in Figure 14, is modeled by the equation

$$
R C \dot{x}=-x+u
$$

where $x$ is the voltage across the capacitor $C$, and the input is given by

$$
u= \begin{cases}-K\left(x-\mu x_{\tau}\left(1-x_{\tau}\right)\right), & x_{\tau} \in\left(0_{-}, 1\right), \\ -K x, & \text { elsewhere. }\end{cases}
$$




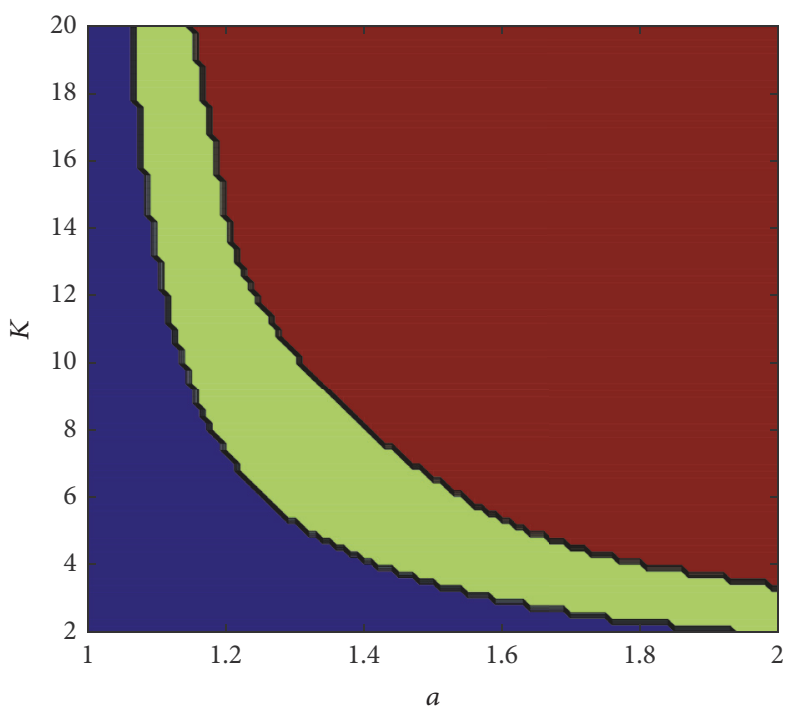

FIGURE 11: Equilibrium (blue), periodic (green), and nonperiodic and chaotic (red) behavior of system (27) with the triangular map (30).

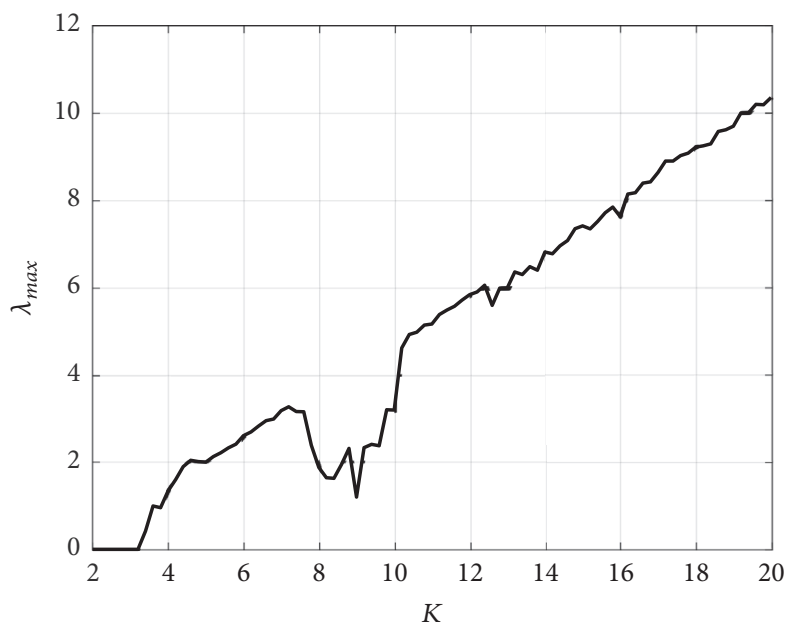

FIGURE 12: Maximum Lyapunov exponent of system (27) with the triangular map (30).

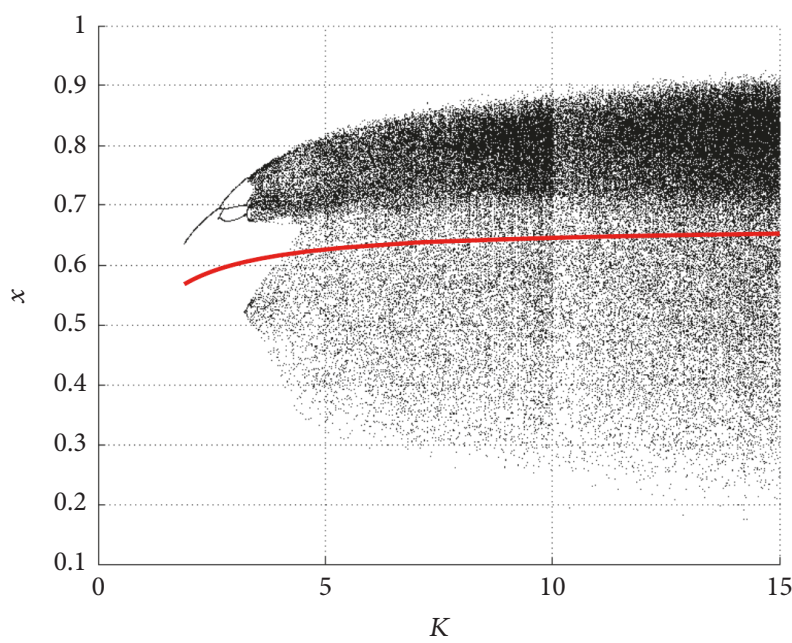

FIGURE 13: Bifurcation diagram and location of unstable equilibrium point of system (27) with the triangular map (30).
The input signal $u$ is generated using Simulink $\odot$, and the interface used between the computer and the circuit is the data acquisition card CLP1104 from dSPACEO.

By choosing the component values $R=1 K \Omega$ and $C=$ $1 \mu F$ the time constant $R C=1 \mathrm{mS}$ is obtained, which scales the dynamics of the circuit (31) by a factor $K_{p}=1 / R C=10^{3}$; that is,

$$
\dot{x}=K_{p}(-x+u) .
$$

This system has the same structure as the example of Section 5.1 (logistic map). The analysis and the application of Theorem 7 are similar and it is possible to calculate all the parameters to satisfy assumptions (AS1-3). Note that, in real cases like this one, a time scaling is needed to apply the results described here.

In what follows we describe the results obtained using $\mu=4.5$ and $\tau=0.1 \mathrm{~s}$. The gain $K$ takes several values to show different behaviors of the circuit. Figure 15 shows the 


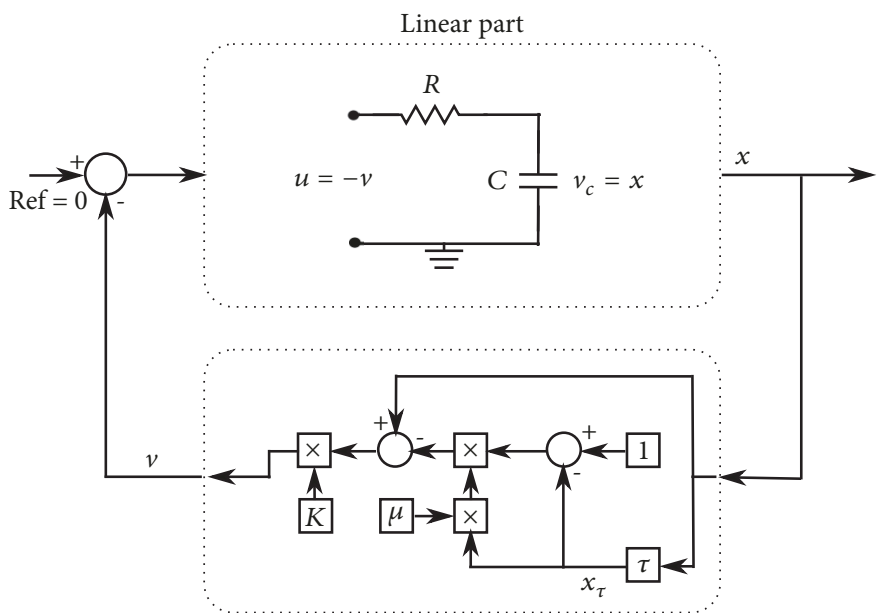

Nonlinear part

FIgURE 14: RC-circuit.

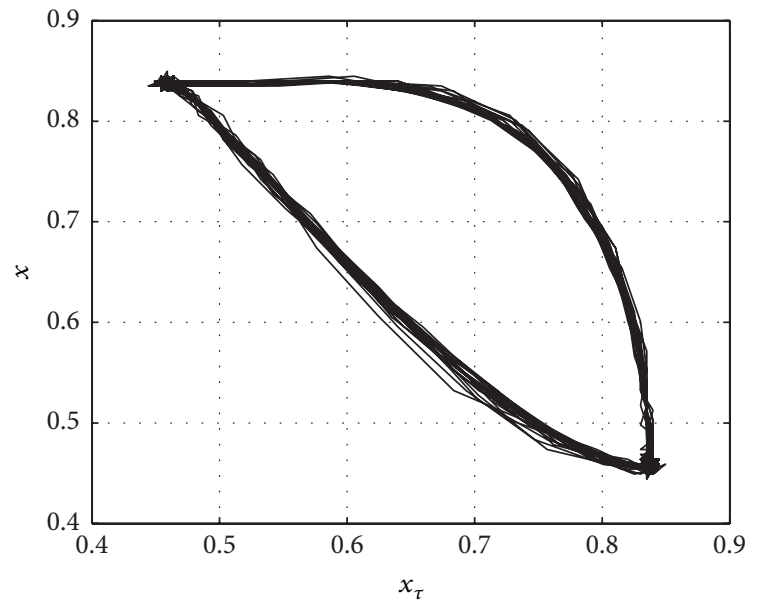

(a) $K=3$

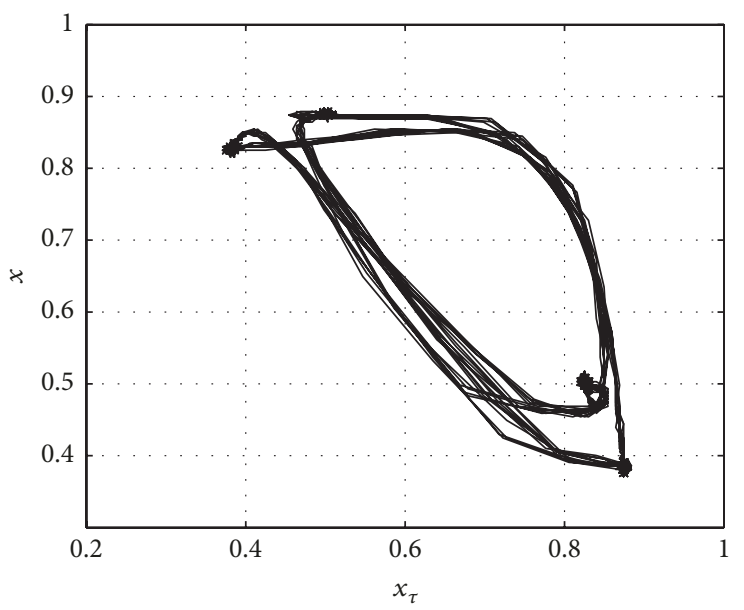

(b) $K=3.5$

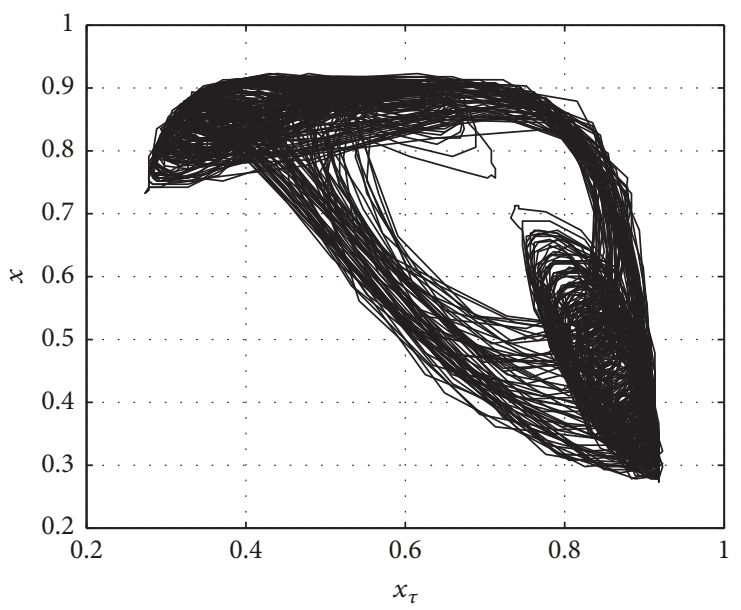

(c) $K=4.5$

FIGURE 15: Different behavior for circuit (31), with $\tau=0.1 \mathrm{~s}, \mu=4.5$. 


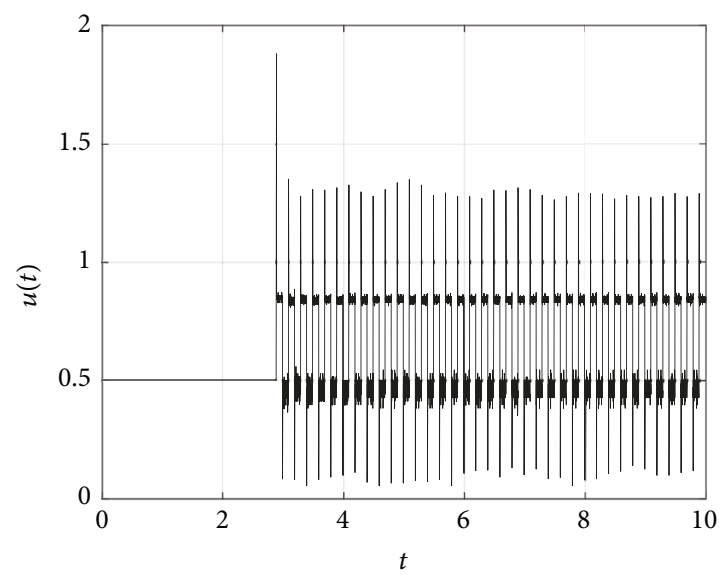

(a) $K=3$

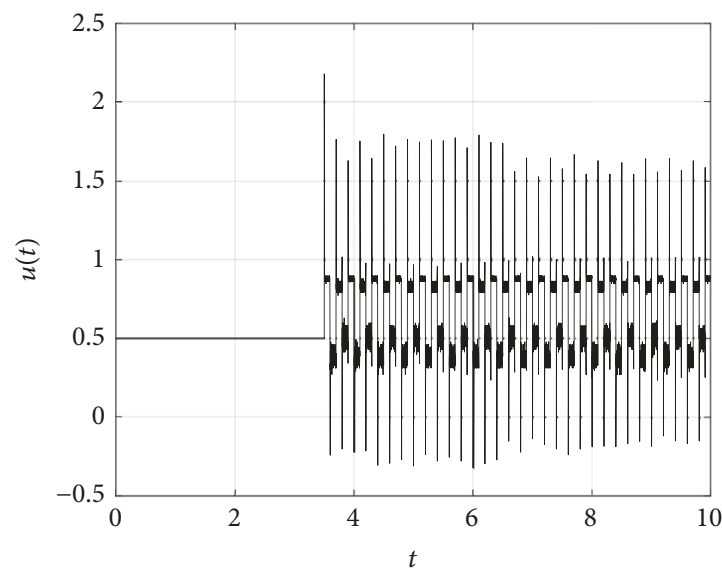

(b) $K=3.5$

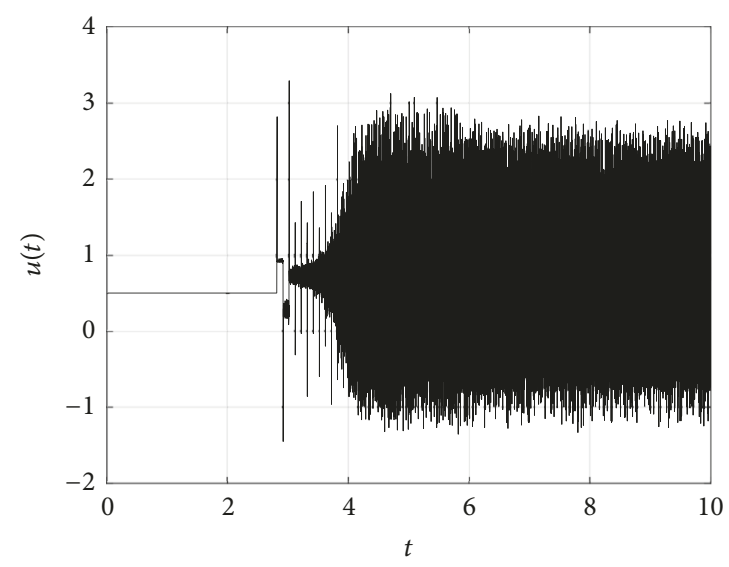

(c) $K=4.5$

Figure 16: Control signals for different $K$ values of the circuit.

responses for several values of parameter $K(3,3.5$, and 4.5). Figure 16 shows the input signal applied to the circuit. Note that the magnitude of this signal lies well inside the voltage range managed for the $\mathrm{D} / \mathrm{A}$ interface $( \pm 5 \mathrm{~V})$.

\section{Conclusions}

In this paper a systematic procedure to induce periodic and chaotic oscillations in simple, first-order, continuoustime systems via a feedback function depending on the delayed state has been proposed. It has been shown that an oscillatory behavior can be produced by imposing a passivity condition on the open-loop system, tuning some parameter to force the equilibrium points to be unstable and guarantee semipassivity of the closed-loop system. Several examples to illustrate the technique were described, with which a great variety of oscillatory behavior can be observed. A physical example built with a simple electronic circuit that displays a similar behavior to the logistic map has also been included.

\section{Conflicts of Interest}

The authors declare that there are no conflicts of interest regarding the publication of this paper.

\section{Acknowledgments}

This work has been partially supported by the National Council for Science and Technology of Mexico.

\section{References}

[1] Y. Kuang, Delay Differential Equations with Applications in Population Dynamics, Academic Press, Providence, RI, USA, 1993.

[2] M. Basso, R. Genesio, and A. Tesi, "Controlling chaos in a CO2 laser," in Proceedings of the European Control Conference (ECC '97), Brussels, Belgium, July 1997.

[3] M. J. Ogorzalek, "Design and implementation of chaos control systems," in Controlling Chaos and Bifurcations in Engineering Systems, G. Chen, Ed., pp. 45-69, CRC Press, Boca Raton, FL, USA, 2000.

[4] P. Celka, "Theory and experiments on nonlinear time-delayed feedback systems with application to chaos control," in Controlling Chaos and Bifurcations in Engineering Systems, G. Chen, Ed., pp. 233-253, CRC Press, Boca Raton, FL, USA, 2000.

[5] X. Yu, Y. Tian, and G. Chen, "Time delayed feedback control of chaos," in Controlling Chaos and Bifurcations in Engineering Systems, pp. 255-274, CRC Press, Boca Raton, FL, USA, 2000. 
[6] J. Milton and P. Jung, Eds., Epilepsy as a Dynamic Disease, Springer, Berlin, Germany, 2003.

[7] L. M. Pecora and T. L. Carroll, "Synchronization of chaotic systems," Chaos, vol. 25, no. 9, pp. 097611.1-097611.12, 2015.

[8] P. Snaselova and F. Zboril, "Genetic algorithm using theory of chaos," Procedia Computer Science, vol. 8, pp. 316-325, 2015.

[9] T. Yang and L. O. Chua, "Chaotic digital code-division multiple access (CDMA) communication systems," International Journal of Bifurcation and Chaos, vol. 7, no. 12, pp. 2789-2805, 1997.

[10] G. Chen, "Chaos: control and anticontrol," IEEE CSS Newsletter, pp. 1-3, 1998.

[11] G. Chen, Ed., Controlling Chaos and Bifurcations in Engineering Systems, CRC Press, Boca Raton, FL, USA, 2000.

[12] G. E. Zheng-Ming, C.-M. Chang, and Y.-S. Chen, "Anti-control of chaos of single time-scale brushless DC motor," Philosophical Transactions of the Royal Society A: Mathematical, Physical \& Engineering Sciences, vol. 364, no. 1846, pp. 2449-2462, 2006.

[13] S. Liu, X. Yu, and S. Zhu, "Study on the chaos anti-control technology in nonlinear vibration isolation system," Journal of Sound and Vibration, vol. 310, no. 4-5, pp. 855-864, 2008.

[14] D. M. Curry, "Practical application of chaos theory to systems engineering," Procedia Computer Science, vol. 8, pp. 39-44, 2012.

[15] Y. Luo, Q. Liu, X. Che, and L. Li, "Damped Least-Square Method Based on Chaos Anti-Control for Solving Forward Displacement of General 6-6-Type Parallel Mechanism," International Journal of Advanced Robotic Systems, vol. 10, no. 4, p. 186, 2013.

[16] Y. Luo, Q. Liu, and X. Che, "Mathematical programming method based on chaos anti-control for the solution of forward displacement of parallel robot mechanisms," International Journal of Advanced Robotic Systems, vol. 10, 2013.

[17] G. R. Chen and D. J. Lai, "Feedback anticontrol of discrete chaos," International Journal of Bifurcation and Chaos, vol. 8, no. 7, pp. 1585-1590, 1998.

[18] X. F. Wang and G. Chen, "On feedback anticontrol of discrete chaos," International Journal of Bifurcation and Chaos, vol. 9, no. 7, pp. 1435-1441, 1999.

[19] X. F. Wang and G. Chen, "Chaotifying a stable LTI system by tiny feedback control," IEEE Transactions on Circuits and Systems I: Fundamental Theory and Applications, vol. 47, no. 3, pp. 410-415, 2000.

[20] T. Y. Li and J. A. Yorke, "Period three implies chaos," The American Mathematical Monthly, vol. 82, no. 10, pp. 985-992, 1975.

[21] K. Pyragas, "Continuous control of chaos by self-controlling feedback," Physics Letters A, vol. 170, pp. 421-428, 1992.

[22] K. Pyragas and A. Tamaševičius, "Experimental control of chaos by delayed self-controlling feedback," Physics Letters A, vol. 180, no. 1-2, pp. 99-102, 1993.

[23] Y.-C. Tian and F. Gao, "Adaptive control of chaotic continuoustime systems with delay," Physica D: Nonlinear Phenomena, vol. 117, no. 1-4, pp. 1-12, 1998.

[24] J. K. Hale and W. Huang, "Period doubling in singularly perturbed delay systems," Journal of Differential Equations, vol. 114, pp. 1-23, 1994.

[25] S.-N. Chow, J. K. Hale, and W. Huang, "From sine waves to square waves in delay equations," Proceedings of the Royal Society of Edinburgh, Section: A Mathematics, vol. 120, no. 3-4, pp. 223-229, 1992.

[26] Z. Artstein and M. Slemrod, "On singularly perturbed retarded functional-differential equations," Journal of Differential Equations, vol. 171, no. 1, pp. 88-109, 2001.
[27] H. Lu and Z. He, "Chaotic behavior in first-order autonomous continuous-time systems with delay," IEEE Transactions on Circuits and Systems I: Fundamental Theory and Applications, vol. 43, no. 8, pp. 700-702, 1996.

[28] X. F. Wang, G. Chen, and X. Yu, "Anticontrol of chaos in continuous-time systems via time-delay feedback," Chaos, vol. 10, no. 4, pp. 771-779, 2000.

[29] J. Alvarez, "Inducing periodic oscillations and chaos in continuous systems via time-delay controllers," in Proceedings of the Control of Oscillations and Chaos Conference (COC'00), pp. 217220, St. Petersburg, Russia, June 2000.

[30] X. F. Wang, "Generating chaos in continuous time systems via feedback control," in Chaos Control, G. Chen and X. Yu, Eds., LNCIS 292, pp. 179-204, Springer Verlag, 2003.

[31] T. S. Zhou, G. R. Chen, and Q. G. Yang, "A simple time-delay feedback anticontrol method made rigorous," Chaos, vol. 14, no. 3, pp. 662-668, 2004.

[32] T. R. Kim, Y. H. Joo, J. B. Park, and G. Chen, "Anticontrol of chaos for a continuous-time Takagi-Sugeno fuzzy system via local time-delay feedback," International Journal of Bifurcation and Chaos, vol. 15, no. 12, pp. 3883-3894, 2005.

[33] Q. Y. Sun, H. G. Zhang, and Y. Zhao, "Generating Li-Yorke chaos in a stable continuous-time T-S fuzzy model via time-delay feedback control," Chinese Physics B, vol. 19, no. 7, pp. 070512/1070512/9, 2010.

[34] T. Zhou, G. Chen, and Q. Yang, "n-scroll chaotic attractors from a first-order time-delay differential equation," Chaos, vol. 17, pp. $1-8,2007$.

[35] A. C. Luo, Periodic Flows to Chaos in Time-Delay Systems, Springer, 2017.

[36] C. H. Moog, R. Castro-Linares, M. Velasco-Villa, and L. A. Marquez-Martinez, "The disturbance decoupling problem for timedelay nonlinear systems," Institute of Electrical and Electronics Engineers Transactions on Automatic Control, vol. 45, no. 2, pp. 305-309, 2000.

[37] J. K. Hale and S. M. Verduyn Lunel, Introduction to FunctionalDifferential Equations, Springer, New York, NY, USA, 1993.

[38] O. Arino, M. Hbid, and E. A. Dads, Delay Differential Equations and Applications, vol. 205, Springer, 2006.

[39] T. Insperger and G. Stépán, Semi-Discretization for Time-Delay Systems: Stability and Engineering Applications, vol. 178 of Applied Mathematical Sciences, Springer Science \& Business Media, New York, NY, USA, 2011.

[40] H. Khalil, Nonlinear Systems, Prentice-Hall, 1996. 


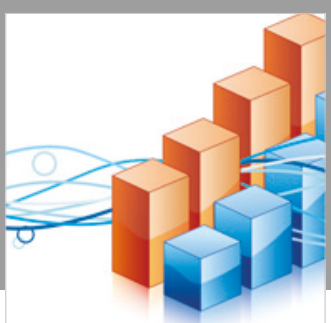

Advances in

Operations Research

\section{-n-m}
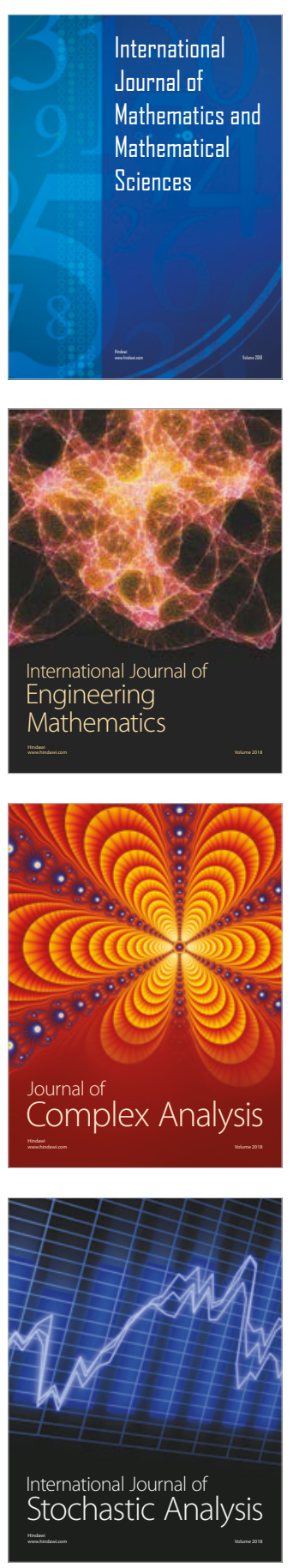
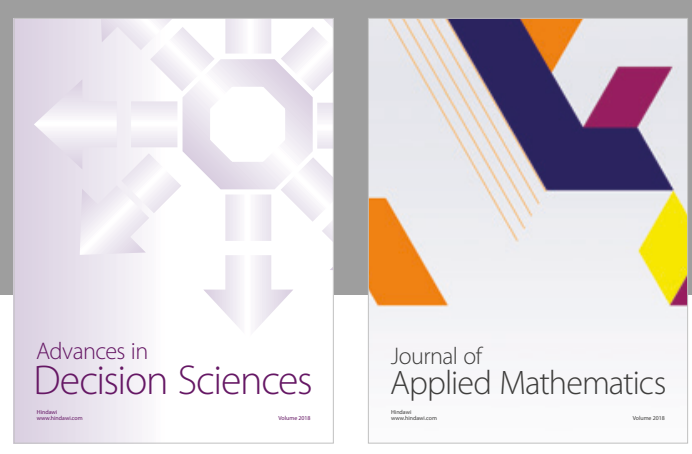

Journal of

Applied Mathematics
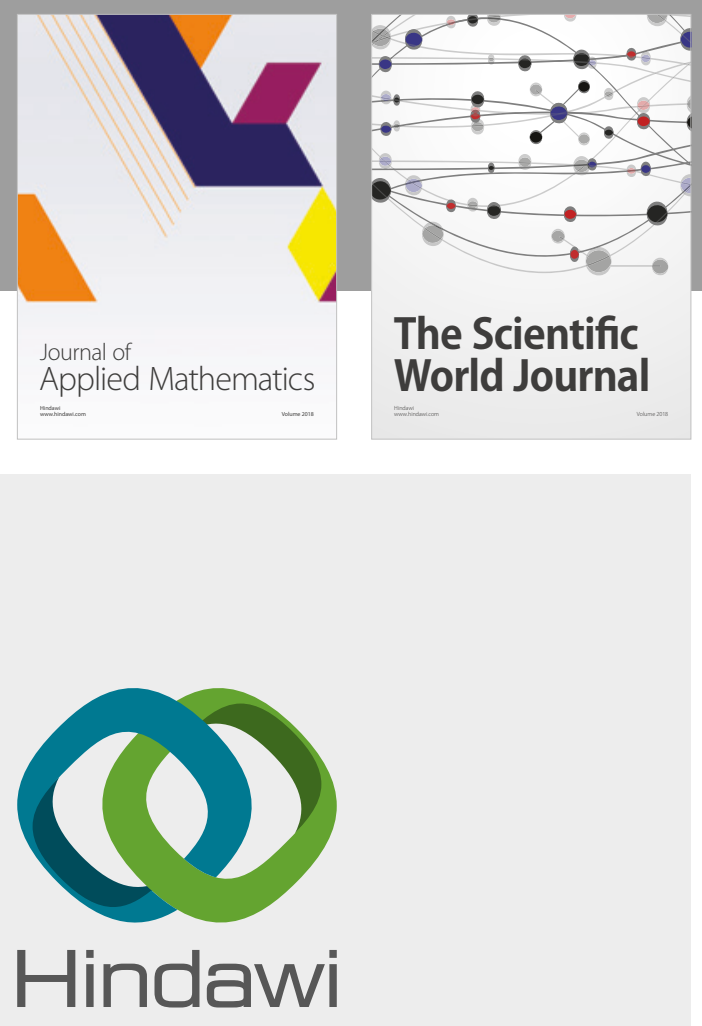

Submit your manuscripts at

www.hindawi.com

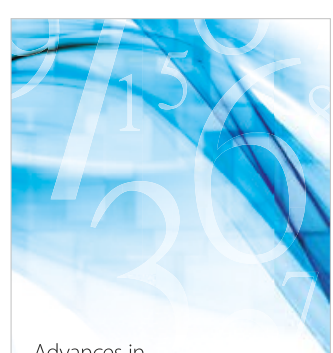

Advances in
Numerical Analysis
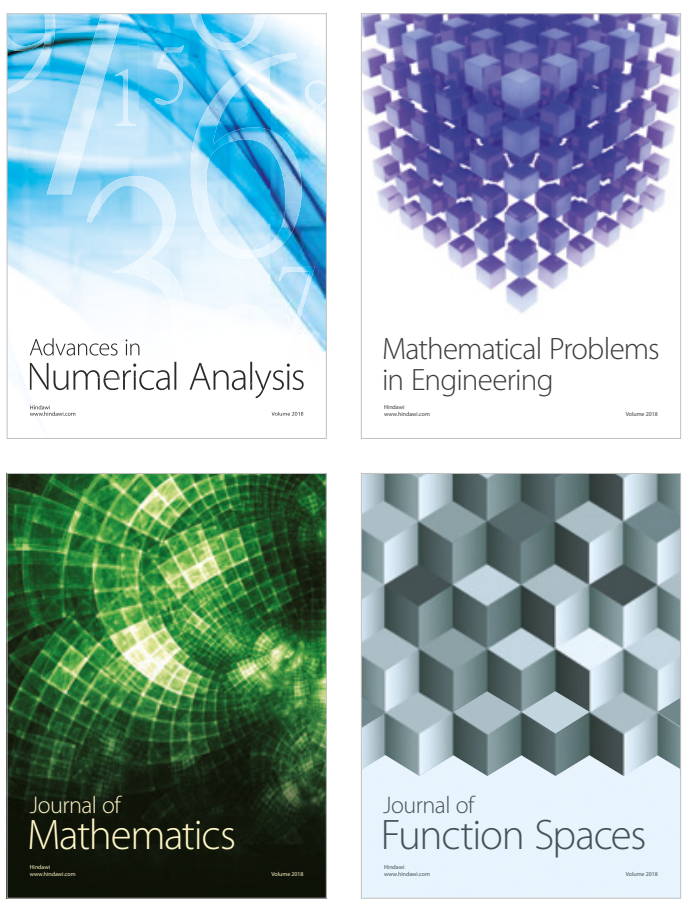

Mathematical Problems in Engineering

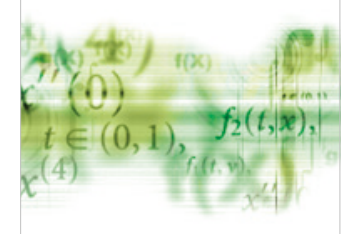

International Journal of

Differential Equations

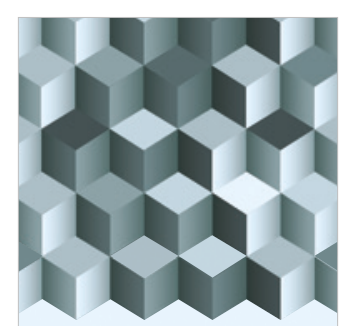

Journal of

Function Spaces
The Scientific

World Journal

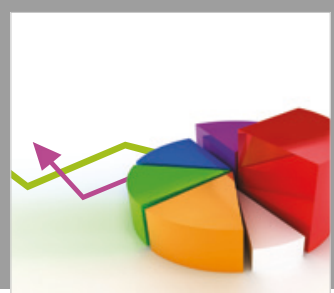

Journal of

Probability and Statistics
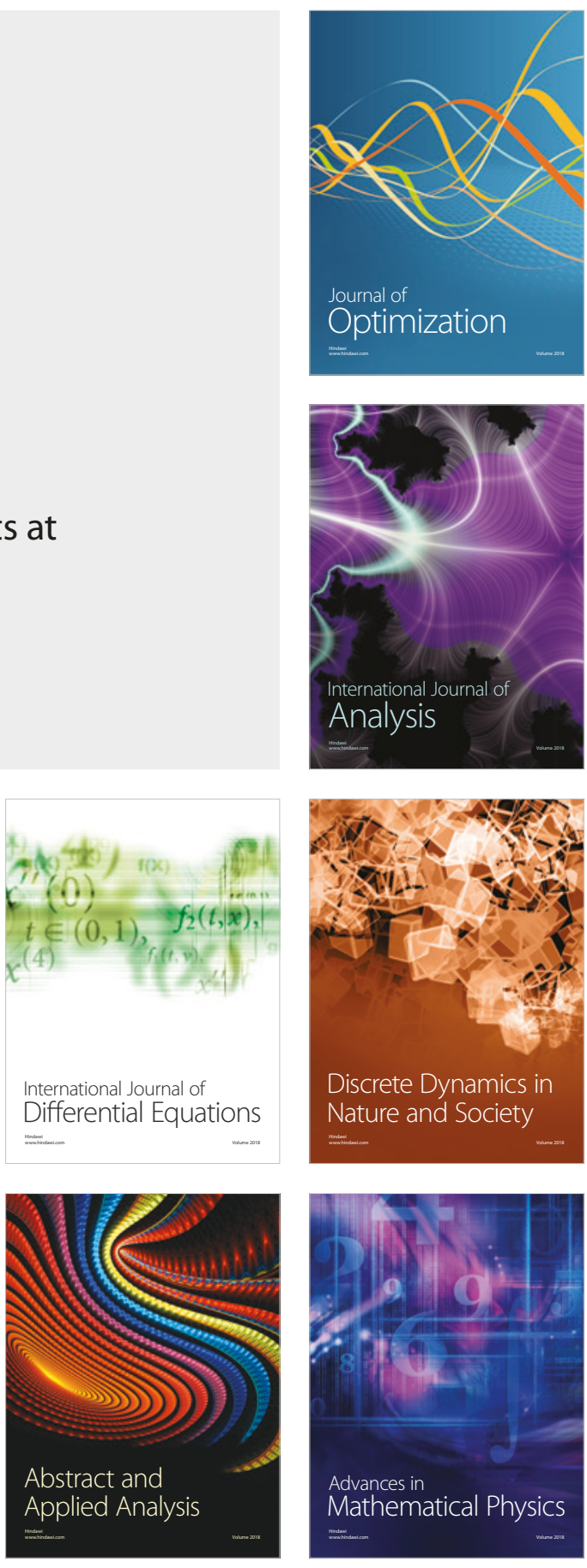